\title{
Accuracy, precision, and quality control of enzyme assays
}

\author{
D. W. MOSS
}

From the Department of Chemical Pathology, Royal Postgraduate Medical School, London

Improvements in standards of analytical accuracy and precision are now firmly established goals in clinical biochemistry. A number of factors have contributed to this. Among them are the appreciation of the value of sequential studies of changes in levels of plasma constituents during the course of an illness, provided that these changes can be distinguished reliably from the inherent variability of the analytical procedures. Furthermore, increased accuracy will lead to a higher degree of standardization of clinical laboratory data: this is important in view of the growing mobility of populations and the likelihood that a patient will attend more than one hospital. Improvements in analytical practice have themselves served to indicate the need for still greater accuracy and precision, in that they have shown the clinical significance of quite minor biochemical changes. Most clinical chemistry laboratories now operate quality control programmes routinely, and the comparison of the analytical performance of different laboratories by interchange of specimens of known composition is increasing. However, enzyme assays have so far benefited less than other procedures from advances in methods of quality control. This is largely due to the physicochemical nature and functional characteristics of enzymes themselves. Efforts to improve the quality of analysis have largely been directed towards monitoring and improving within-batch and between-batch precision and improving accuracy. In enzyme analysis the principal difficulty is to find a satisfactory method by which the accuracy of a determination can be assessed. This paper discusses possible approaches to the calibration or standardization of enzyme assays, as well as problems encountered in assessing precision.

Enzymes are catalysts, and in almost all cases are assayed by measurements of their catalytic activities, ie, the extent to which they increase the rate of a given chemical reaction. This is dependent on the conditions under which it is measured, including the concentration of the reactants, temperature, and $p H$. The sensitivity of the rate of a chemical reactio $\vec{n}$ to changes in such conditions is generally much greater in the presence of an enzyme than in itso absence. As enzyme activities depend on the cons ditions under which they are determined, a particular unit of enzyme activity must depend on the particular method of assay. It is not practicable at present to determine absolute quantities or molar concerno tration of an enzyme except in a very few cases. given enzyme is rarely present in a sufficiently pure form for its concentration to be estimated bB के determination of protein. Also, while a knowleges of the specific molecular activity of the enzyme undef the conditions of assay would allow activity measume? ments to be translated into molar concentrations such information is scanty; even if this were availablo estimations of activity under strictly defined cone ditions would still be necessary. Since estimation ô enzymes for clinical purposes must continue $t \sigma$ depend on activity measurements, two approaches to standardization are possible. The first is the fomulation of agreed standard methods of assay which define corresponding enzyme units. The second is the provision of reference preparations of enzymes t $\bar{\alpha}$. which are assigned agreed values of activity; these preparations may then be used as calibration standards in secondary methods of enzyme estimae tion, or as quality control preparations to asses analytical performance. The two approaches are not mutually exclusive; indeed, the existence of reference. enzyme preparations presupposes that these have been assigned values on the basis of activity detern mination, unless a completely arbitrary choice of values is accepted. Therefore, standard methods of analysis and the availability of reference preparations of enzymes are both desirable in clinical enzymology? At present agreement on standard methods of analys sis, at least for the more frequently estimated enzymes seems to be the more easily attainable goal and ons that is being actively pursued by specialist groups. 
The Nature and Origin of Diversity in Enzyme Assay Procedures

The prime consideration in the design of an enzyme assay method is that the amount of enzyme present should be the only variable which affects the reaction rate. This condition is more likely to be met if the assay procedure ensures optimal substrate concentration, $p \mathrm{H}$, temperature, etc, and thus many of the modifications that have from time to time been made to assay methods have been motivated by the desire to achieve optimal conditions. Unfortunately, it has not always been appreciated that such modifications almost inevitably result in an alteration in the enzyme activity as measured by the method.

The use of suboptimal conditions does not necessarily invalidate an assay procedure, particularly if only one type of enzyme or isoenzyme is to be measured. For example, the ratio of two enzyme activities will be independent of substrate concentration provided that the two samples have similar Michaelis curves, ie, the same Michaelis constant, $\mathrm{K}_{\mathrm{m}}$. However, when the Michaelis constants of the two samples differ, the ratio of the two activities becomes increasingly dependent on substrate concentration as this falls progressively below saturating levels (Fig. 1). One source of variation in $\mathrm{K}_{m}$ from one enzyme sample to another is a difference in their isoenzyme composition, such as is well recognized in the case of lactate dehydrogenase isoenzymes. It should be emphasized, however, that changes in $\mathrm{K}_{\mathrm{m}}$ of the magnitude of those shown for the alkaline phosphatase samples in Fig. 1 can arise even when no change in the isoenzyme is involved. Alkaline phosphatase $\mathbf{K}_{\mathbf{m}}$ values are affected by the composition and degree of purity of the enzyme solution (Moss, Campbell, AnagnostouKakaras, and King, 1961) so that different serum samples do not necessarily show the same $\mathbf{K}_{\mathbf{m}}$ values even when they contain the same isoenzymes. The Michaelis constant of alkaline phosphatases is also very markedly dependent on $p \mathrm{H}$ in the region of $p \mathrm{H10}$, at which phosphatase assays are usually carried out (Fig. 2), so that inadequate control of $p \mathrm{H}$ can cause variation in the apparent $\mathrm{K}_{\mathrm{m}}$. Vesell and others have pointed out the effect of temperature on the shape of the Michaelis curves of lactate dehydrogenase isoenzymes (Vesell and Pool, 1966). Similar considerations apply to other variables in the enzyme assay system, notably the effects of $p \mathrm{H}$ and temperature. The shape of the $p \mathrm{H}$-activity curve of an enzyme in the region of the optimum is largely governed by the ionization characteristics of functional groups in the active centre, but at more extreme $p \mathrm{H}$ values factors such as enzyme stability

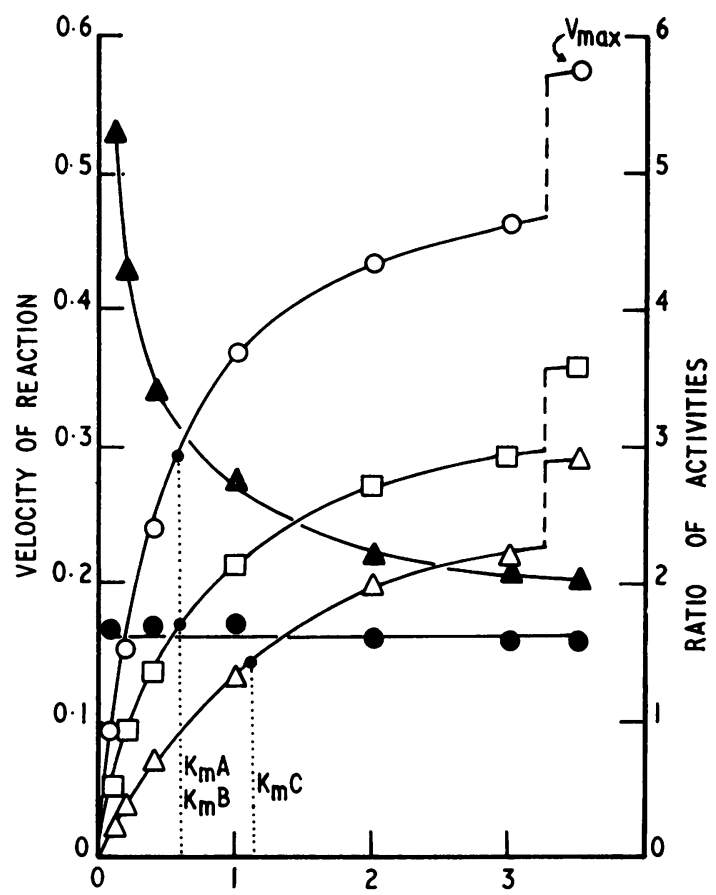

[SUBSTRATE] $\mathrm{mM}$

Fig. 1 Relationship between reaction velocity ( $\mu$ mols substrate hydrolysed per min per ml of enzyme solution) and substrate concentration (mM p-nitrophenyl phosphate) at pH 10.0 for three specimens of human alkaline phosphatase. $A(O)$ and $B(\square)$ are both liver phosphatase and have the same $K_{m}$ value; $C(\triangle)$ is intestinal phosphatase with a slightly different $K_{m}$. The ratio of the activities of $A$ and $B(O)$ is independent of the substrate concentration at which the activities are measured, but that of $A$ and $C(\Delta)$ is increasingly dependent on substrate concentration as this is decreased.

come into play to an extent which may affect enzyme concentration. Thus, the ratio of the activities of two samples of the same enzyme is independent of the $p \mathrm{H}$ of measurement when this is close to the $p \mathrm{H}$ optimum, but may not be so when sub optimal $p H$ values are used. Figure 3 shows this effect for alkaline phosphatase. The several variables in the assay system may be so interrelated that it may not be possible to achieve optimal values for each and some degree of compromise may be necessary. This is especially true when fixed-time assays are used or when a single assay system is to be applied to samples which contain mixtures of isoenzymes in varying 


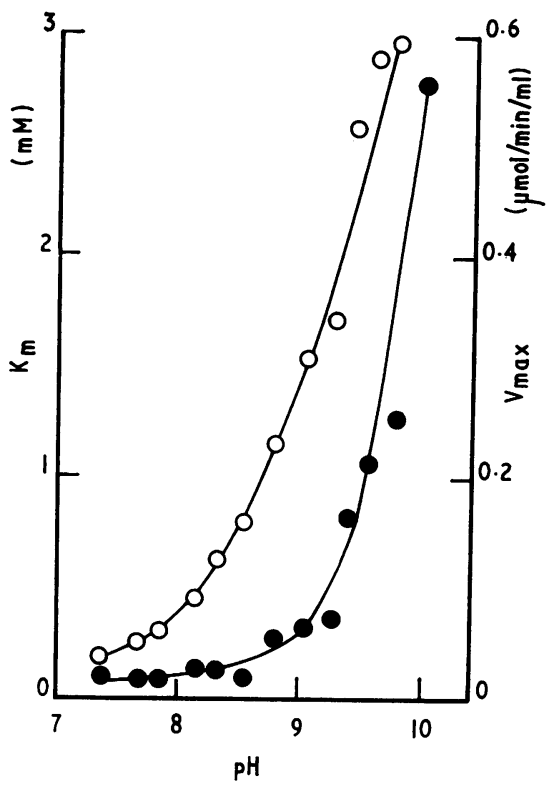

Fig. 2 Relationship between $K_{m}$ value (O; $m M p$ -

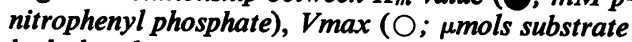
hydrolysed per min per ml of enzyme solution) and $\mathrm{pH}$ measurement for human bone alkaline phosphatase, showing the marked dependence of $K_{m}$ on $\mathrm{pH}$ in the region of $\mathrm{pH} 10$ (data from Moss, 1969).

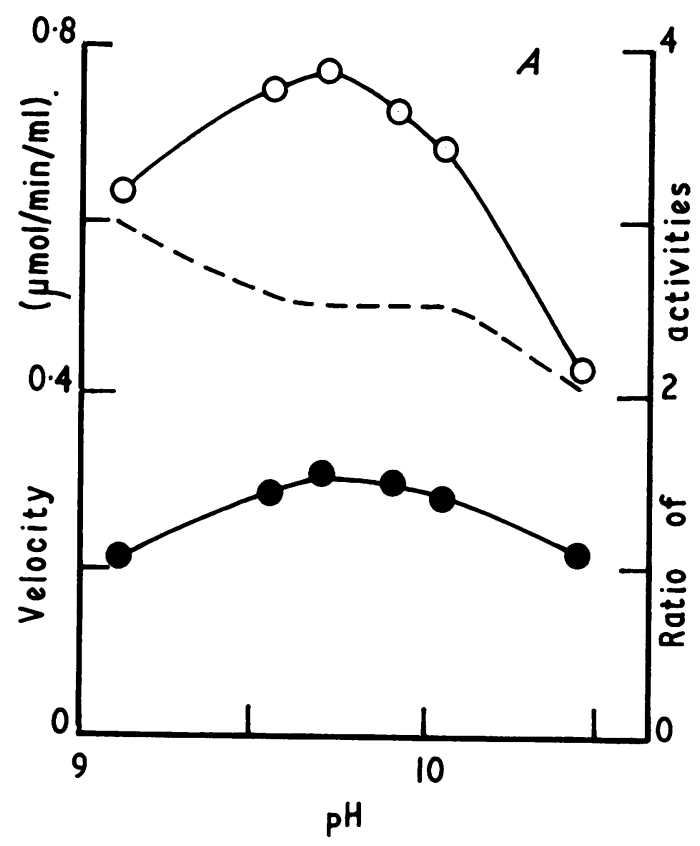

Fig. 3a.

Fig. 3 A Activity-pH curves for two samples o, human intestinal alkaline phosphatase $(O$ and $O)$. The ratio of the activities (broken line) of the two samples is independent of $\mathrm{pH}$ only over a narrow range around the $\mathrm{pH}$ optimum.

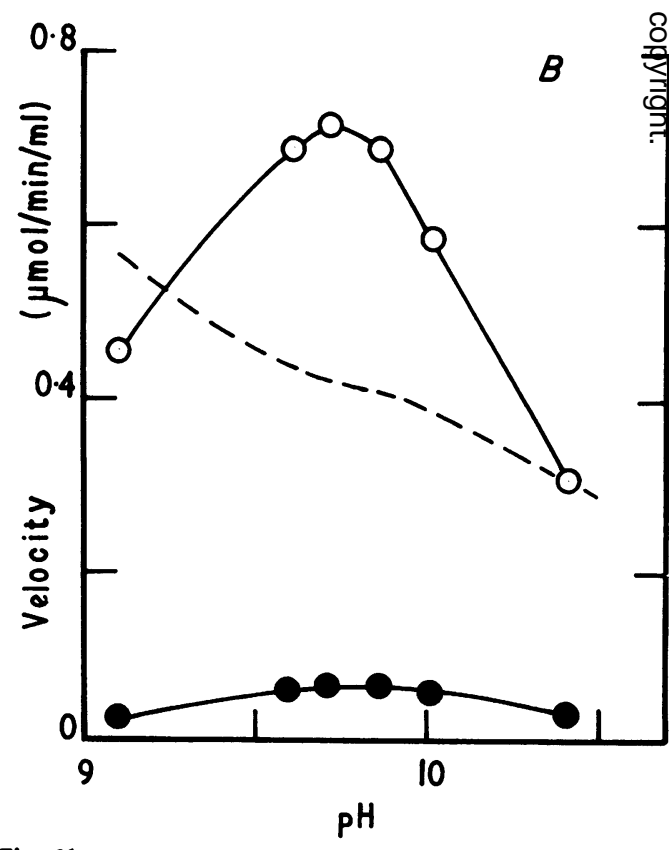

Fig. 3b.

B The same for two liver alkaline phosphatase samples here the range over which the ratio is independent of $\mathrm{pH} ?^{\text {? }}$ is even narrower. (Substrate in both $A$ and $B, 4 m M$ p-nitrophenyl phosphate.) 
proportions, as is the case with human lactate dehydrogenase in serum, since optimal conditions for the activity of isoenzyme 1 are significantly different from those for isoenzyme 5(Gay, McComb, and Bowers, 1968).

Another reason for the modification of methods of enzyme estimation is to improve analytical sensitivity or specificity of the methods that are employed to determine the amount of chemical change taking place. The scope for this type of modification is greater when enzyme specificity is low so that the nature of the substrate can be varied, as exemplified by the many substrates that have been used in the assay of alkaline phosphatase; but even without changing substrate, sensitivity can be increased by changing the method of estimating the product. The extent to which the results of unmodified enzyme assays can be compared with those obtained after modification depends on the nature of the changes which have been made. If these do not include alterations in the conditions under which the enzyme acts, or in the nature of the substrate, the results should be fully comparable. For example, the method chosen for the estimation of phenol released from phenyl phosphate by phosphatase does not affect the level of enzyme activity. However, when the conditions of hydrolysis are changed, eg, by changing the substrate from phenyl phosphate to p-nitrophenyl phosphate or merely by altering the $\mathrm{pH}$ or nature of the buffer, the level of enzyme activity is also altered. The foregoing discussion of the complex relationships that obtain between the many variables in an enzyme assay system indicates the difficulty that may then be encountered in relating units derived from the modified method to those from the original procedure.

Not all alterations to methods of enzyme estimation are intentional, however. When procedures have been long established a gradual drift from the specified protocol often takes place and may result in considerable modification of the method. The significance of the results consequently undergoes change.

The foregoing arguments may be summarized as follows. First, the variables in all enzyme assay procedures must be rigidly specified and controlled. Enzyme activities should not be reported in terms of units which imply a particular method unless that method has been strictly followed. It is desirable that the methods on which enzyme units in common use are based should be re-examined and re-stated to remove ambiguity. Secondly, since many older methods are clearly unsatisfactory in the light of recent advances in enzymology, new definitive assay methods are needed for enzymes of importance in clinical enzymology.

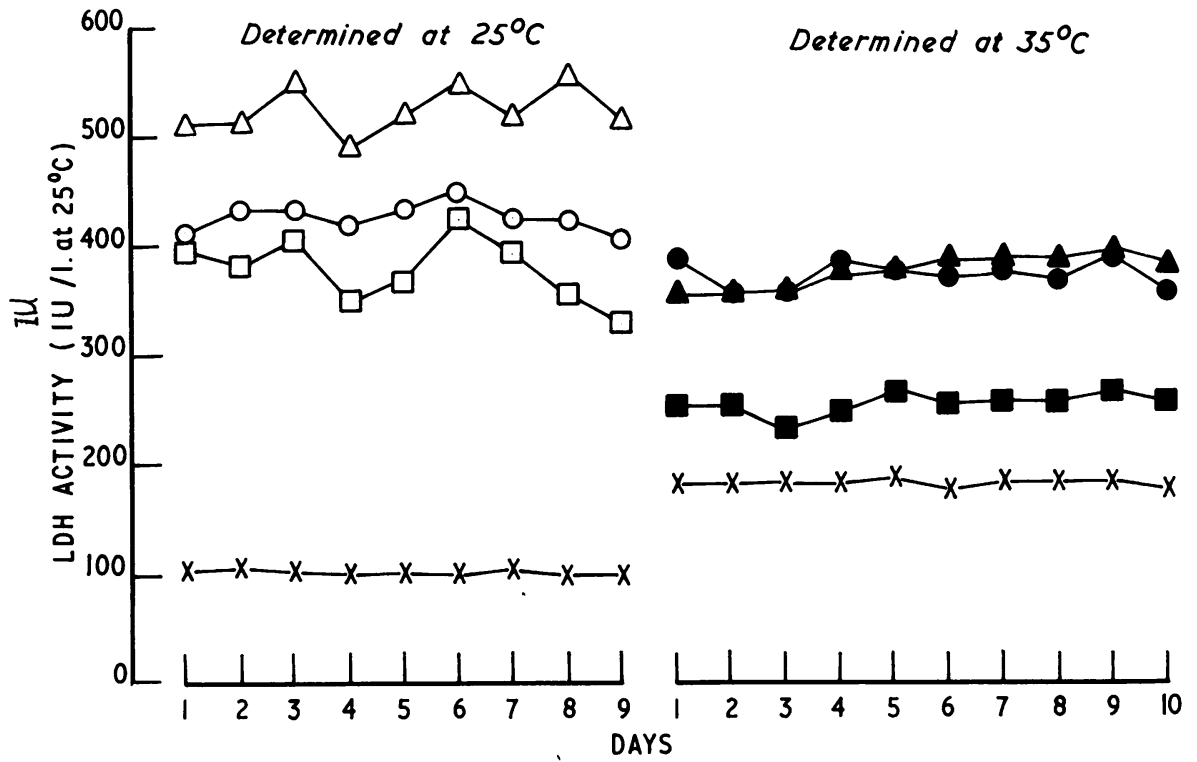

Fig. 4 Daily quality-control data for the spectrophotometric determination of serum lactate dehydrogenase. $X$, pooled serum; $\bigcirc, \triangle, \square, O, \Delta, \square$, reconstituted lyophilized materials. Considerable between-batch and within-batch variation is apparent at $25^{\circ}$, particularly at higher enzyme activities. A change to $35^{\circ}$ is followed by an improvement in precision. 


\section{Factors Affecting the Design of New Reference Methods}

The theoretical advantages of obtaining a continuous record of enzyme reaction rates can now be realized as a result of the greater availability of recording spectrophotometers. Newer reference methods of enzyme assay should be based on this principle wherever possible, rather than on a fixed-time or twopoint basis. Apparatus designed to combine some degree of automation with kinetic analysis is now becoming available ${ }^{1}$ and the clinical enzymologist should state clearly those features which he expects to find in such instruments. Specifications for optical components, reagent dispensers, and sample transfer systems will, in general, be similar to those required in other automated equipment. However, one feature of central importance is the provision of a thermostatically controlled reaction cuvette, so that it is possible to control the temperature at which measurements are to be made.

Fixed-time assays have traditionally been carried out at $37^{\circ} \mathrm{C}$ but with the introduction of kinetic assays $25^{\circ} \mathrm{C}$ became widely accepted as a standard temperature. However, whereas temperatures such as $37^{\circ} \mathrm{C}$ that are well above the ambient temperature almost everywhere can be accurately maintained by simple, inexpensive thermostats controlling a heating

${ }^{1}$ See contribution by Dr Skillen on page 31 .

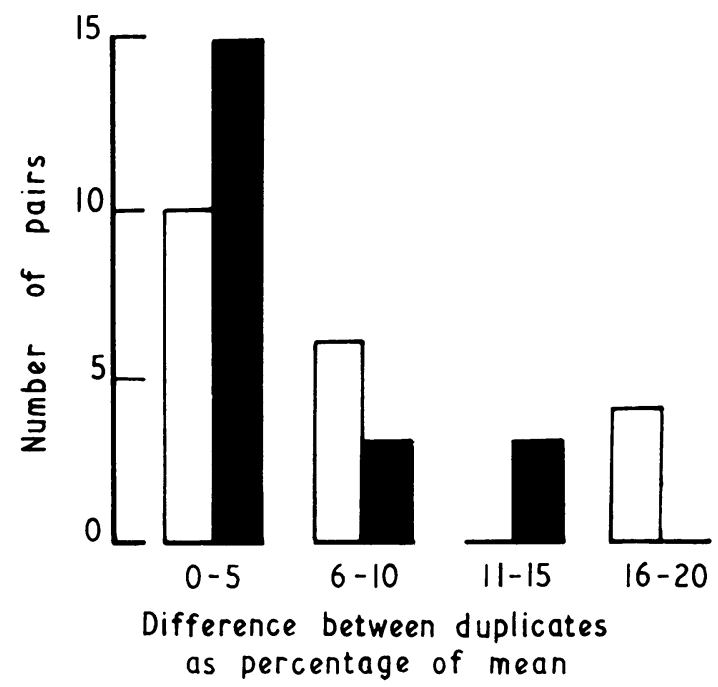

Fig. 5 Differences between duplicate serum lactate dehydrogenase estimations, expressed as a percentage of the mean value for each pair, determined spectrophotometrically at $25^{\circ} \mathrm{C}$ (open bars) and $35^{\circ} \mathrm{C}$ (solid bars). circuit, $25^{\circ} \mathrm{C}$ is close to, or even below, the ambie $\frac{\rho}{\overline{\bar{n}} \mathrm{t}}$ temperature in many laboratories so that this simp form of temperature control becomes inefficient or even totally ineffective. The Enzyme Commission of the IUB has therefore recommended an increase the standard temperature to $30^{\circ} \mathrm{C}$. Nevertheless, some instruments now available are set to operate at even higher temperatures, eg, $35^{\circ} \mathrm{C}$, and require $\mathrm{c}^{\mathrm{a}}$ differential of at least $8^{\circ} \mathrm{C}$ above the ambie temperature for temperature control to be effective. Figures 4 and 5 illustrate the great improvement $\mathrm{m}$ the quality of results following a change of tempersture for a kinetic analysis of lactate dehydrogenase from $25^{\circ}$ to $35^{\circ} \mathrm{C}$ with a consequent improvement $\overrightarrow{\dot{\phi}}$ control of temperature. However, before accepting higher reaction temperature for reasons of analytical convenience the clinical enzymologist will wish to be assured that the selected temperature is suitable other respects also: for example, that factors such $\overrightarrow{\mathrm{AS}}$ instability of enzymes or isoenzymes during the pres incubation or measuring periods do not affect the results obtained. If the use of higher temperatures is unacceptable instruments which incorporate bod heating and cooling cycles will become necessar, and, in this case, apparatus with a liquid incubation bath or circulation system may prove more readily adaptable than those depending on electrical heatiing of metal racks or air chambers.

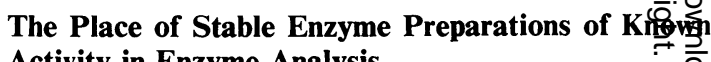
Activity in Enzyme Analysis

While standardization of assay methods will cont $\stackrel{8}{8}$ bute greatly to uniformity in clinical enzymology it nevertheless desirable that stable enzyme prepar: tions of known activity should be available fer determining the relative accuracy of results obtained in different laboratories. A number of preparation; usually freeze-dried sera or mixed tissue extracts, ape available to which values of activity of variogs enzymes have been assigned but, however usef these preparations have been, it is generally agreed that they have not been accepted with the same degree of confidence that has been bestowed on quality-control preparations for other, non-enz matic constituents. This is because clinical enzyrizologists have often experienced difficulty in obtaining results which agree with the assigned values for the control preparations, even though the laboratoryso own quality control programme suggests the्t analytical methods are operating satisfactoril. Doubts are therefore cast on the stability of reference preparations, and on the accuracy of thepr original calibration.

Some examples may help to illustrate these point Figures 6 and 7 show that, in assays of two differe 


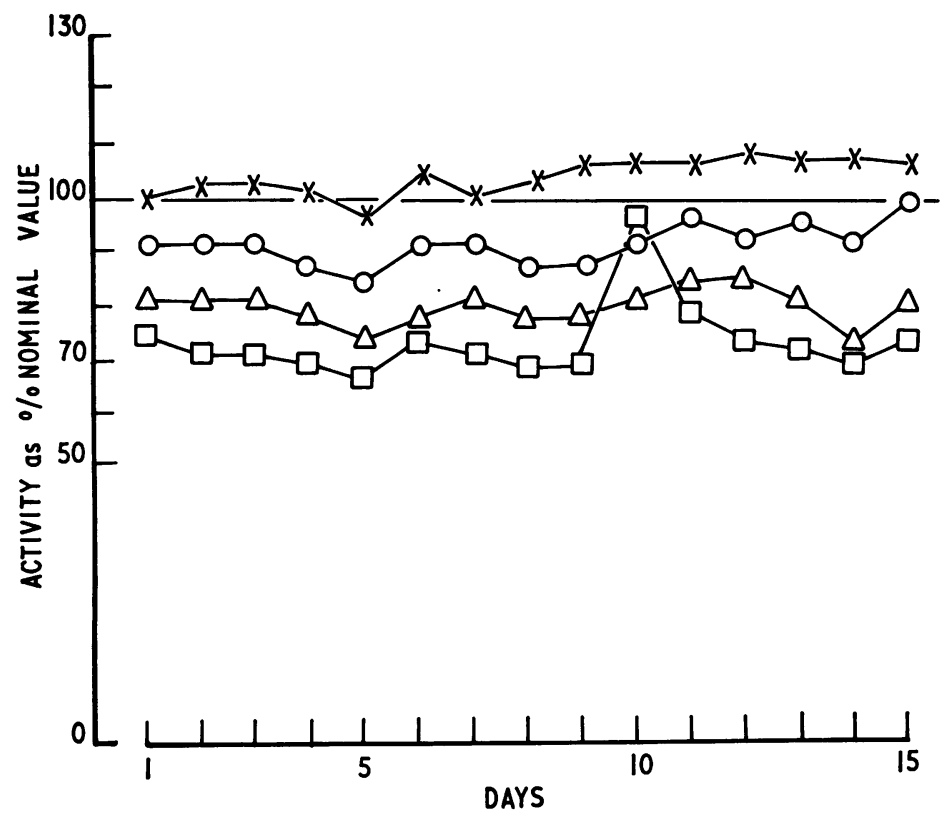

Fig. 6 Daily quality control data for determination of serum alkaline phosphatase by a colorimetric phenylphosphate method on the AutoAnalyzer. $\bigcirc, \triangle, \square$, reconstituted lyophilized commercial control preparations, freshly prepared each day. Activities are expressed as a percentage of the respective nominal values. $X$, pooled serum. The method was calibrated and the activity of the pooled serum determined by $a$ manual King-Armstrong procedure.

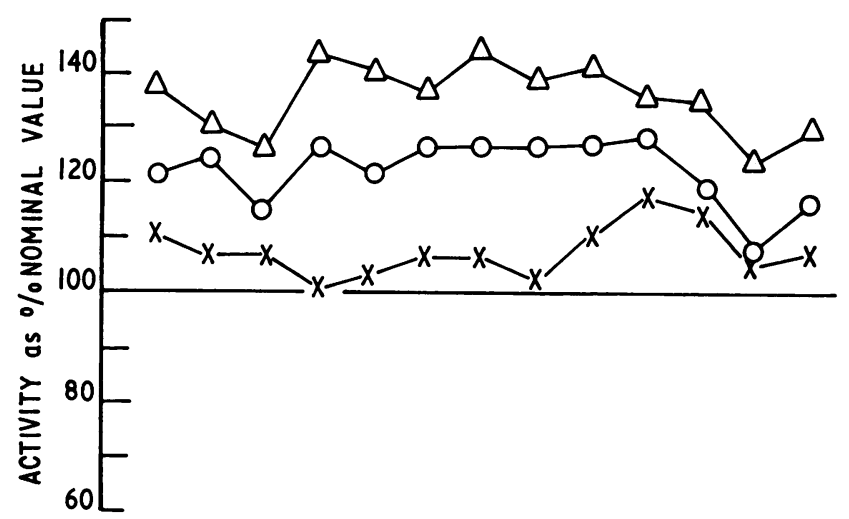

Fig. 7 Daily quality control data for a colorimetric aspartate aminotransferase method on the AutoAnalyzer. The procedure was calibrated by reference to one commercial lyophilized material, and results for two others $(O, \triangle)$ are expressed as a percentage of their respective nominal values. $X$, pooled serum.

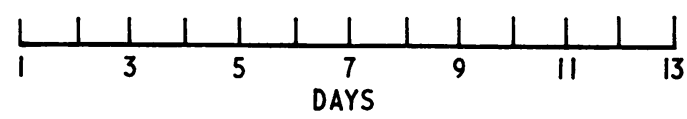

enzymes, control sera supplied from outside the laboratory fail to reproduce their assigned activities. The deviation from the nominal value is a random one and does not seem to be due to a systematic error on the part of the user's laboratory, since if this were the case the control materials should all show values of more or less the same percentage of their nominal values. Dobrow and Amador (1970) have recently published data which also show that nominal values often cannot be reproduced. Within a particular batch of control material day-to-day reproducibility was generally good, with a few exceptions, and this is in agreement with previous reports (eg, Hanok and Kuo, 1968).

Different batches of material from a single source did not show a consistent deviation from the nominal value. It is not possible to decide on the basis of the present data whether the discrepancies are due to deviations in assay procedures between the calibrating and using laboratories, or whether a relatively 
rapid change to a different level of activity takes place after calibration, followed by a longer period of stability. In this respect recent observations that the enzymatic activity of reconstituted lyophilized sera, as well as that of frozen serum pools, increases over a considerable period of time following reconstitution or thawing may be relevant (B. Brojer and D. W. Moss, unpublished data).

Whatever the explanation, a user would not feel confident in accepting these preparations at their nominal values of activity without re-calibrating them himself and may prefer to rely on frozen serum pools prepared in the laboratory or repeated analysis of specimens of patients' serum for monitoring within-batch or between-batch variation.

\section{Required Properties of a Standard or Reference Enzyme Preparation}

The most important characteristic of a standard enzyme preparation is stability. Recent studies of the three-dimensional structures of protein molecules have emphasized still further the dependence of catalytic activity on the integrity of the molecular architecture of enzyme molecules. Derangement of this structure, ie, denaturation, leads to loss of activity. When relatively concentrated and pure solutions of enzymes or other proteins are available, the occurrence of denaturation can be detected by changes in a number of physical properties of the solution. However, for the dilute solutions of enzymes in use as controls or calibration standards in enzyme assay, with their admixture of other proteins, such measurements are not possible and the only detectable consequence of denaturation is a decline in catalytic activity. In the absence of assured stability there is thus no way in which a low result arising from faults in analysis can be distinguished from one due to deterioration of the reference preparation. Increased stability of enzyme reference preparations may require changes in procedures of production and storage and even in the chemical constitution of the enzymes themselves, so that before these possibilities are discussed it is necessary to consider which of the properties of the enzymes must be preserved and which characteristics can be permitted to undergo change, and to what extent, in the pursuance of increased stability. If enzyme reference preparations are intended to provide a means of testing the accuracy of a particular assay and to compare the results obtained in one laboratory with those obtained elsewhere, there is no reason why the reference catalyst should be identical in all respects with the enzyme that the assay method is designed to measure. The reference catalyst may therefore be an enzyme of similar function but of markedly different stability characteristics prepared from a non-human sourc $\stackrel{\varrho}{\overline{\bar{e}}}$ It may be an enzyme that has been modified chengcally to increase its resistance to denaturation at the expense of some alteration in properties. It may, indeed, not be an enzyme at all but some other for of catalyst. For example, the cyclohexamyloses described by Bender and others (Hennrich and Cramer, 1965; Bender, van Etten, Clowes agf Sebastian, 1966) possess esterase activity towards number of organic esters, and, although their degree of activity in this respect does not compare with that of true enzymes, compounds of this nature may hase properties that make them suitable for testing the accuracy of reaction rate measurements

However, if the purpose of enzyme reference preparations is to control the routine operation $9 f$ assay methods, or to calibrate one method in terms of another, then the reference sample should be closely similar as possible to the unknown samples under test. Neglect of this principle can result hin error if factors operating in the test system affect the enzymes in the unknown and reference samples differently. For example, a change which results di suboptimal conditions, and therefore in reducet activities, for the unknown enzyme samples may net have the same effect on the reference preparation if this is of different isoenzymic composition properties, and instances in which preparatione of non-human alkaline phosphatase have failed equately to control estimations of the human en have been reported (MacWilliam, Moody, and Sills, 1967; Bowers, Kelley, and McComb, 1967). Wheres differential effect of this nature exists, its results ca become particularly significant when the referenge enzyme preparation is used in the calibration of one enzyme assay method by comparison with a different procedure. A conversion factor established for tue reference preparation may not be valid if samples containing a qualitatively different type of enzyne are analysed. Anomalies of this nature are mofe likely to arise when enzymes of low specificity, such as alkaline phosphatase, are being estimated, when a change in method may imply a change in the substrate. Human placental alkaline phosphatase is a valuable source of reference material for pho 8 phatase determinations, because of its remarkabe stability to heat and other denaturing agentso However, placental alkaline phosphatase differs in important respects from the liver and bone is enzymes of which the alkaline phosphatase in serum usually consists. Among these differences a variations in the relative rates at which different substrates are hydrolysed (Moss, 1969; Wof, Dinwoodie, and Morgan, 1969). Placental phos" phatase, and also intestinal phosphatase, are relatively less effective in their action on some 
chromogenic substrates such as p-nitrophenyl phosphate than on non-aromatic phosphate esters such as $\beta$-glycerophosphate. There are also differences in relative rates of breakdown of $\mathrm{p}$ nitrophenyl phosphate and thymolphthalein monophosphate by liver and intestinal phosphatases. These differences imply that if, for example, a conversion factor for translating p-nitrophenyl phosphatase units into thymolphthalein monophosphatase units is established by comparisons based on samples which contain intestinal phosphatase, this factor will not be valid when samples containing liver or bone phosphatases are analysed. It follows that some substrates provide a more sensitive assay than others for certain isoenzymes.

\section{Possible Approaches to Enzyme Stabilization}

As already mentioned, the constancy of the activity of an enzyme depends on the preservation of the three-dimensional structure of its molecules. Also important is the protection of vulnerable amino acid side chains which may have an important role in the catalytic mechanism. Reactive groups on the exterior of the molecule probably play only a small part in determining overall conformation but modification of external charged residues may nevertheless affect stability. Ways of increasing the stability of enzyme molecules may be divided into those designed to protect the molecules from the access of denaturing or inactivating factors, and those designed to increase the resistance of molecules to these factors (Fig. 8).

Examples of the first approach, ie, the prevention of the access of inactivating factors, are the addition of substances to the enzyme preparation to ensure a favourable environment, eg, an acid $p \mathrm{H}$ for acid phosphatase or reducing conditions for enzymes that depend for their activity on sulphydryl groups. The enclosure of enzyme molecules within semipermeable membranes (Chang, 1964) also increases enzyme stability, presumably by the same mechanism. Changes in the resistance of enzyme molecules to inactivation as a consequence of the chemical modification of reactive groups are examples of the second approach. For instance, acetylation of some human alkaline phosphatases increases their resistance to denaturation at alkaline $p \mathrm{H}$ values but decreases it at more acid values (Moss, 1970). A similar effect is noted when enzyme molecules are attached to an insoluble matrix, commonly by their terminal and side-chain amino groups (Silman and Katchalski, 1966). This latter procedure probably also increases stability by making the enzyme molecule more rigid, but it must be remembered that slight conformational changes appear to be an essential feature of the mechanism of enzyme catalysis and, while these intramolecular movements are probably of a lower order of magnitude than those involved in denaturation, the need for some degree of molecular flexibility will ultimately limit the extent to which the enzyme molecule can be stiffened. Similar considerations apply to the introduction of additional cross-links between adjacent segments of the polypeptide chain. Covalent crosslinkages, except for disulphide bridges, do not seem to be a major feature in stabilizing the structures of native enzyme molecules which depend for their

\section{ENZYME STABILIZATION}

\section{BY INCREASING RESISTANCE}

TO DENATURATION

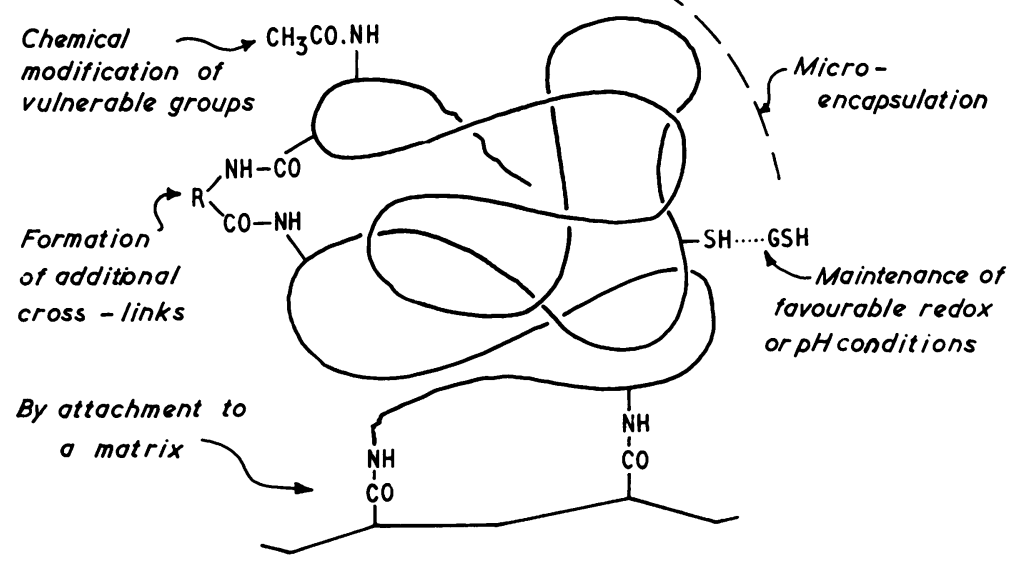

Fig. 8 Diagrammatic representation of possible approaches to stabilization of enzyme molecules for the preparation of reference or control samples. 
conformation mainly on the existence of multiple hydrophobic interactions and hydrogen-bonding. However, the attachment of enzymes to soluble matrices seems to be an approach to stabilization which merits exploration.

These several ways of increasing the stability of enzyme preparations differ in the degree to which the catalytic properties of the enzymes are changed. Such changes must be taken into account when modified enzymes are put to use in standardization and control of enzyme assays, for the reasons discussed earlier. The preservation of an acid $p \mathrm{H}$ or reducing conditions in the environment should not significantly alter functional characteristics, but the chemical modification of reactive groups may be expected to alter catalytic properties to an extent which will depend on the relationship between these groups and the active centre. In the example of the acetylation of human alkaline phosphatases referred to previously, the modified enzymes are unchanged in their Michaelis constants and not significantly different from the native forms in substrate specificity, as judged by their relative orthophosphatase and pyrophosphatase activity. There is a slight alkaline shift in the $p \mathrm{H}$ optimum of intestinal alkaline phosphatase but this is due to the changes in $p \mathrm{H}$ stability characteristics rather than to a modification of the active centre; it would thus be expected to be more pronounced in assay methods depending on an extended incubation period. Unfortunately, liver phosphatase is affected less than intestinal phosphatase by acetylation, while the increase in stability of the intestinal isoenzyme is not such as to meet the requirements of a reference enzyme preparation.

While the application of these principles to the production of enzymes possessing long-term stability is at the moment in an elementary state, knowledge of enzyme structure is growing rapidly and enzyme technology is consequently also expanding quickly. The prospects for the production of stabilized enzyme preparations of defined characteristics as a result of systematic research programmes therefore are encouraging.

In summary, therefore, two initiatives in the standardization and quality control of enzyme ass $\stackrel{\rho}{\overline{\overline{\mathrm{A}}}}$ are needed, and are beginning to be implementod. The first of these is the re-definition, rationalizati $\overline{\mathrm{gn}^{n}}$, and standardization of enzyme assay procedures, while the second is the provision of stabilized enzy reference preparations. Progress in both thesse directions can be expected to increase the value 0 of clinical enzymology.

References

Bender, M. L., Van Etten, R. L., Clowes, B. A., and Sebastian, J.? (1966). A pictorial description of the 'lock and key' the $\overrightarrow{a y}$ J. Amer. chem. Soc., 88, 2318-2319.

Bowers, G. N., Jr., Kelley, M. L., and McComb, R. B. (1987). Precision estimates in clinical chemistry. I. Variabilityof analytic results in a survey reference sample related to the us? of a non-human serum alkaline phosphatase. Clin. Chem., 1,3, 595-608.

Chang, T. M. S. (1964). Semipermeable microcapsules. Science, 46 , 524-525.

Dobrow, D. A., and Amador, E. (1970). The accuracy of commerifal enzyme reference sera. Amer. J. clin. Path., 53, 60-67.

Gay, R. J., McComb, R. B., and Bowers, G. N., Jr. (1968). Optimum reaction conditions for human lactate dehydrogenase isoenzymes as they affect total lactate dehydrogenase activity. Clin. Chem., 14, 740-753.

Hanok, A., and Kuo, J. (1968). The stability of a reconstituted ser for the assay of fifteen chemical constituents. Clin. Chem., $\mathbf{d} 4$, 58-69.

Hennrich, N., and Cramer, F. (1965). Inclusion compounds. 18. The catalysis of the fission of pyrophosphates by cyclodextrinca model reaction for the mechanism of enzymes. J. Amer. Soc., 87, 1121-1126.

MacWilliam, K. M., Moody, A. H., and Silk, J. (1967). Variatronth alkaline phosphatase results using the method of Bessey, and Brock. Clin. chim. Acta, 17, 514-515.

Moss, D. W. (1969). Bioshemical studies on phosphohydrole্টse isoenzymes. Ann. N.Y. Acad. Sci., 166, 641-652.

Moss, D. W. (1970). Chemical modification of alkaline phosphatas. The effects of amino-group reagents on the electrophorefic mobilities and activities of phosphatases from several human tissues. Enzymologia, 39, 319-330.

Moss, D. W., Campbell, D. M., Anagnostou-Kakaras, E., and King. E. J. (1961). Characterization of tissue alkaline phosphatases and their partial purification by starch-gel electrophores. Biochem. J., 81, 441-447.

Silman, I. H., and Katchalski, E. (1966). Water-insoluble derivatises of enzymes, antigens, and antibodies. Ann. Rev. Biochem., 873-908.

Vesell, E. S., and Pool, P. E. (1966). Lactate and pyruvate conc대trations in exercised ischemic canine muscle: relationship. of tissue substrate level to lactate dehydrogenase isozyme pattef‥ Proc. nat. Acad. Sci. (Wash.), 55, 756-762.

Wolf, M., Dinwoodie, A., and Morgan, H. G. (1969). Comparison alkaline phosphatase isoenzymes activity using five stand@d methods. Clin. chim. Acta, 24, 131-134. 